\title{
Optimizing Throughput for Cryo-EM Data Collection in Drug Discovery
}

\author{
Stephen Mick, Sahil Gulati and Christopher Booth
}

Gatan, United States

Direct electron detection camera technology for transmission electron microscopy has provided a dramatic improvement in the quality of images that can be acquired in cryo-electron microscopy (cryo-EM). Improvement in image quality is one of the key factors that has led to the resolution revolution ${ }^{1}$ so that reconstructions of biological macromolecules are now commonly resolved to a resolution better than 3.5 Angstroms.

Although this resolution revolution opens new opportunities for cryo-EM to solve key problems in molecular biology ${ }^{2}$, it remains a challenge to increase imaging throughput while maintaining the highest image quality. This is primarily due to the numerous inter-related microscope and camera parameters that need to be carefully optimized to balance between imaging efficiency and image quality. Optimization of these parameters is of critical importance for biopharmaceutical research since both image quality and throughput must be maximized ${ }^{3}$.

Weis and Hagen provide a good assessment of microscope configuration trade-offs and discuss both pixel size and magnification ${ }^{4}$, but there are several other key considerations related to the camera and data collection software that impact cryo-EM data quality and throughput. These considerations include optimization of coincidence loss, electron dose rate, field of view, dose fractionation, motion correction, data storage format, energy filter slit width where a filtered-camera system is used, and managing both the sites at which to collect data and the data acquisition queue for automated data collection.

As an example, increasing electron dose rate will increase imaging efficiency (i.e. the total area that can be imaged in one hour), but this carries the risk of reducing image quality due to an increase in coincidence loss (i.e. the percentage of missed electron events during electron counting). Table 1 shows imaging efficiency that can be achieved for two different dose rates given an exposure that results in a total dose of $30 \mathrm{e}^{-} / \AA^{2}$, and Figure 1 shows the relationship between coincidence loss and imaging efficiency using the K3 camera with a nominal pixel size of $1 \AA^{2}$. Given this trade-off, careful consideration is required when choosing a dose rate to balance between imaging efficiency and image quality.

Table 1: Imaging efficiency for data collection with two different dose rates. In both cases, the data is shown for a K3 direct detection camera using a nominal $1 \AA^{2}$ pixel, 2 second total exposure, and total dose of $30 \mathrm{e}^{-} / \AA^{2}$.

\begin{tabular}{|c|l|c|c|}
\hline Dose Rate $(\mathrm{e}-/ \mathrm{px} / \mathrm{sec})$ & Imaging Area $\left(\mu \mathrm{m}^{2}\right)$ & Imaging Rate (images/hr) & Imaging Efficiency $\left(\mu \mathrm{m}^{2} / \mathrm{hr}\right)$ \\
\hline 15 & & & 153 \\
\hline 25 & 0.235 & 650 & 198 \\
\hline
\end{tabular}

Using the $\mathrm{K} 3$ direct detection camera with Latitude $\mathrm{S}$ automation software as an example configuration, this presentation will discuss the trade-offs for the camera and acquisition software and provide practical guidance to maximize image quality and throughput under realistic imaging conditions. 


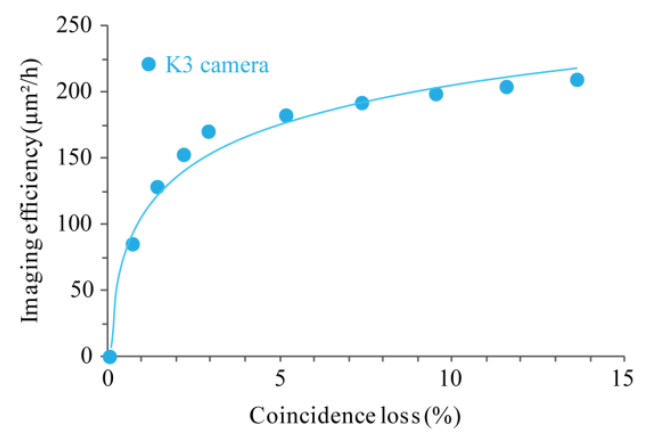

Figure 1. Figure 1: Imaging efficiency plotted against coincidence loss for a K3 direct detection camera. Datapoints are calculated using a nominal $1 \AA 2$ pixel, 2 second total exposure, and total dose of $30 \mathrm{e}$-/ $\AA 2$.

References

[1] Kühlbrandt, W., (2014), The Resolution Revolution, Science, 343 (6178), 1443-1444, doi:10.1126/science.1251652.

[2] Hanske, J., Sadian, Y., Müller, C.W., (2018), The cryo-EM resolution revolution and transcription complexes, Current Opinions in Structural Biology, 52:8-15, doi.org/10.1016/j.sbi.2018.07.002.

[3] Maia de Oliveira, T., van Beek, L., Shilliday, F., Debreczeni, J.E., Phillips, C., (2020), Cryo-EM: The Resolution Revolution and Drug Discovery, SLAS Discovery: Advancing the Science of Drug Discovery, 26(10), 17-31, doi.org/10.1177/2472555220960401.

[4] Weis, F., Hagen, W., (2020), Combining high throughput and high quality for cryo-electron microscopy data collection, Acta Crystallographica Section D: Structural Biology, 76(8), 724-728, doi.org/10.1107/S2059798320008347. 